\title{
Efficacy of extended clomifene citrate regimen in comparison with gonadotropins in clomifene citrate-resistant women with polycystic ovary syndrome
}

\section{Mahmoud Fathy Hassan*}

Department of Obstetrics \& Gynecology, Faculty of Medicine, Ain Shams University, Cairo, Egypt

Received: 1 January 2014

Accepted: 11 January 2014

\section{*Correspondence:}

Dr. Mahmoud Fathy Hassan,

E-mail: mahmoudfathy74@yahoo.com

(C) 2014 Hassan MF. This is an open-access article distributed under the terms of the Creative Commons Attribution Non-Commercial License, which permits unrestricted non-commercial use, distribution, and reproduction in any medium, provided the original work is properly cited.

\begin{abstract}
Background: Gonadotropins are successful treatment for women with clomifene citrate (CC)-resistant polycystic ovary syndrome (PCOS). The aim of this study was to test the hypothesis that extended CC treatment may be an alternative to gonadotropins in the management of CC-resistant women with PCOS.

Methods: A randomized controlled trial comprised 200 women with CC-resistant PCOS were allocated to two equal treatment groups. Patients in the CC group were given $100 \mathrm{mg}$ of CC daily starting from the third day of menses for 10 days. Patients in the gonadotropins group were given follitropin-alfa according to step-up regimen starting on the $3^{\text {rd }}$ day of menses. The primary outcome measure was the biochemical pregnancy rate [diagnosed by measuring serum $\beta$-human chorionic gonadotropin ( $\beta$-HCG) 16-days after HCG injection] and clinical pregnancy rate (confirmed by vaginal ultrasound at six-weeks of amenorrhea). Secondary outcomes were: ovulation rate, endometrial thickness at HCG injection, and adverse drug events.

Results: There were no statistically significant differences between the $\mathrm{CC}$ group and the gonadotropins group regarding biochemical pregnancy rates $[21 \%, 24 \%$; respectively, $P=0.735$; relative risk $(\mathrm{RR})=0.88,95 \%$ confidence interval (CI), 0.52-1.47], and clinical pregnancy rate [19\%, 21\%; respectively, $P=0.86$; RR=0.9, (95\% CI, 0.52-1.58)]. No significant difference was displayed regarding ovulation rate, endometrial thickness at HCG, and adverse drug events.

Conclusions: The extended $\mathrm{CC}$ regimen appears to constitute a good alternative to gonadotropins therapy in patients with CC-resistant PCOS. Further multi-center studies are needed to confirm our results and to provide more powerful evidence.
\end{abstract}

Keywords: Clomifene resistance, Gonadotropins, Follitropin-alfa, Polycystic ovary syndrome

\section{INTRODUCTION}

Polycystic ovary syndrome (PCOS) is the most common endocrine disorder in women of reproduction age. The prevalence of PCOS, using different diagnostic criteria, has been reported to be $6.8-18 \% .^{1}$ Clomifene citrate $(\mathrm{CC})$ has been widely used as the standard first line treatment for ovulation induction in women with PCOS for more than four decades. ${ }^{2}$ Although CC treatment will frequently restore ovulation in approximately $80 \%$ of women, those $20 \%$ of women that do not ovulate on the maximal daily dose of $150 \mathrm{mg}$ are referred to as $\mathrm{CC}$ resistant. $^{3} \quad$ Obesity, insulin resistance, and hyperandrogenemia denote the major factors involved in CC resistance; prevent the ovaries from responding to raised endogenous follicle-stimulating hormone (FSH) levels following CC therapy. ${ }^{4}$ In addition, a genetic predisposition was suggested for the $\mathrm{CC}$ resistance. ${ }^{5}$ Alternatives to $\mathrm{CC}$ therapy in $\mathrm{CC}$ resistant PCOS patients include aromatase inhibitors, tamoxifen, insulinsensitizing agents, ovarian drilling, gonadotropins, and in vitro fertilization. ${ }^{6}$ 
The action of $\mathrm{CC}$ is based on raised endogenous FSH that falls again after the typical five days course of therapy is completed. $^{7}$ Meanwhile, ovulation induction using gonadotropins therapy is based on the physiological concept that initiation and maintenance of follicle growth may be achieved by a transient increase in FSH above a threshold dose for sufficient duration to generate a limited number of developing follicles. ${ }^{8}$ There is no evidence of a difference between recombinant $\mathrm{FSH}$, urinary FSH, and highly purified FSH for ovulation induction in CC resistant PCOS women. ${ }^{9}$ However, gonadotropins administration is characterized by the need of intensive ovulation monitoring, increased risk of multiple pregnancy, ovarian hyperstimulation syndrome (OHSS), and high direct and indirect costs. ${ }^{10}$

In the present study, we hypothesized that a prolonged use of $\mathrm{CC}$ for 10 days in ovulation induction might overcome the $\mathrm{CC}$ resistance, and provide comparable treatment outcomes when compared with gonadotropins therapy in CC resistant PCOS patients in the form of biochemical and clinical pregnancy rates as well as the ovulation rate.

\section{METHODS}

We conducted this randomized trial in the Infertility Clinic of a large Governmental Hospital, Dhahran, Saudi Arabia from June 2011 to June 2013. The present study included 200 infertile women with PCOS and with clomifene resistance. All participants were divided equally and allocated either to a prolonged CC (Group 1), or to follitropin-alfa (Group 2) for induction of ovulation. The protocol was approved by the Local Institutional Ethics and Research Committee and a written informed consent was taken from all participants before conducting the study. All the procedures, in this trial were conducted in compliance with the ethical principles for medical research involving human subjects of the World Medical Association (Declaration of Helsinki).

Diagnosis of PCOS was made according to the Rotterdam's criteria when two of three features are present including: oligo-ovulation or anovulation, hyperandrogenism (either clinical and/or biochemical), and the presence of polycystic ovaries. ${ }^{11}$ The clomifene resistance in women with PCOS was defined as failure to ovulate after receiving $150 \mathrm{mg}$ of $\mathrm{CC}$ daily for five days per cycle, for at least three cycles. ${ }^{3}$

A thorough evaluation of all participants was carried out via history taking, physical examination; and routine infertility work up for PCOS patients. The routine infertility work-up performed for all participants included baseline trans-vaginal ultrasound, early follicular serum FSH, luteinizing hormone (LH), and testosterone, homeostatic model assessment of insulin resistance (HOMA-IR), ${ }^{12}$ hysterosalpingogram and husband semen analysis. Husband's semen analysis was considered normal according to WHO 2010 criteria. $^{13}$
Inclusion criteria for participation were: the presence of a written consent, clomifene resistance, age $>16$ years and $\leq 35$ years, normal hysterosalpingogram, and normal semen analysis parameters. Exclusion criteria were baseline ovarian cysts or uterine pathology, infertility due to causes other than PCOS or due to combined factors, and a known allergy to clomifene or follitropin-alfa.

Women, who were meeting the study criteria and consented for participation, were randomly allocated to one arm of the study trial. Group 1 included a woman who received clomifene $100 \mathrm{mg}\left(\right.$ Clomid $^{\circledR}$, SanofiAventis, Paris, France) for 10 days starting on day three of induced or spontaneous cycle. Meanwhile, group 2 included a woman who received follitropin-alfa (Gonal$\mathrm{f}^{\circledR}$, Merck Serono, Geneva, Switzerland) according to the low dose step up protocol, starting on day three of a spontaneous or induced cycle by means of subcutaneous injections of $75 \mathrm{IU} /$ day. If no ovarian response was detected after one week, the daily dose was increased to 112.5 IU for one week, and then to 150 IU. $^{9}$

The randomization list was generated by the study statistician (using the online research randomizer software

http://www.graphpad.com/quickcalcs/index.cfm) using permuted block randomization with block sizes varying from 4 to 8 . The allocation sequence was concealed from the researcher enrolling and assessing participants in sequentially numbered, sealed, and opaque envelopes. Envelopes were opened after the enrolled participants completed all baseline assessments and it was time to assign the intervention. The study was open labeled; thus, women and clinicians were aware of the treatment allocation scheme.

Ovulation induction was monitored by vaginal ultrasound every second to third day starting on day nine of the cycle. Triggering ovulation was made by intramuscular injection of 10,000 IU human chorionic gonadotropin (HCG; Profasi HP ${ }^{\circledR}$, Serono S. A., Geneva, Switzerland) when the leading follicle reached $\geq 18 \mathrm{~mm}$ diameter. Free sexual intercourse was encouraged from the day of HCG administration only in cases when no more than three follicles bigger than $17 \mathrm{~mm}$ were observed; then, HCG injection was not administered and protected sexual intercourse was recommended in order to avoid highorder multiple conception. Biochemical pregnancy was assessed by serum $\beta$-HCG assay 16 days after HCG injection and clinical pregnancy was confirmed by vaginal ultrasound at six weeks of amenorrhea.

The primary outcome measures were the biochemical and clinical pregnancy rates, while the secondary outcomes included the percentage of women succeeded to have mature follicles $\geq 16 \mathrm{~mm}$ in average dimension, endometrial thickness at the time of HCG injection, midluteal serum progesterone and the rate of adverse drug events including OHSS. 
The required sample size was calculated using the PS Power and Sample Size Calculation, version 3.0.43 (Department of Biostatistics, Vanderbilt University, Nashville, TN, USA). The primary outcome measures in this trial were the biochemical and clinical pregnancy rates. On the basis of previous studies, ${ }^{14} 94$ participants were required in each group to detect this difference with a $5 \%$ level of significance ( $\alpha$ error $=0.05$ ) and $80 \%$ power $(1-\beta=0.8)$.

Statistical analysis was performed using the Statistical Package for Social Sciences, version 14.0 (SPSS Inc., Chicago, IL, USA) and GraphPad Prism, version 6 (GraphPad Software Inc., La Jolla, CA, USA). Analysis was performed on an intention-to-treat basis. The Shapiro Wilk test was performed to test the Gaussian distribution of continuous variables. Normally distributed numerical data were presented as mean and standard error of mean. Non-normally distributed data were presented as median and range. Qualitative data were presented as the number and percentage. Normally distributed numerical data were compared with unpaired Student's $t$-test. Non- normally numerical data were compared with the Wilcoxon rank-sum test. Qualitative data were compared using the Fisher exact test. Relative risk (RR) with $95 \%$ confidence interval (CI) was evaluated for the outcome measures of both groups. For all tests, the statistical significance was assumed when $P<0.05$ with two tails.

\section{RESULTS}

A total of 272 women initially recruited to participate in this clinical trial; however only 200 participants were included in the study and randomized into two equal groups (Figure 1). Table 1 demonstrates that the two study groups were comparable as regards factors that may affect the ovulation induction or the pregnancy rate.

There was no statistically significant difference between the two studied groups regarding age, BMI, oligomenorrhea, hirsutism, Waist to hip ratio, prior pelvic surgery, HOMA-IR, basal FSH and LH, testosterone, sperm count and motility, and other baseline characteristics.

Table 1: Baseline characteristics of the study groups.

\begin{tabular}{|c|c|c|c|}
\hline Variable & CC group $(n=100)(\%)$ & Follitropin-alfa group $(n=100)(\%)$ & $P$ value \\
\hline Age (years) & $28.5 \pm 0.37$ & $28.7 \pm 0.41$ & 0.718 \\
\hline BMI $\left(\mathrm{kg} / \mathrm{m}^{2}\right)$ & $28.9 \pm 0.63$ & $28.1 \pm 0.82$ & 0.44 \\
\hline Duration of infertility (years) & $3(2-6)$ & $3(2-5)$ & 0.342 \\
\hline \multicolumn{4}{|l|}{ Type of infertility } \\
\hline Primary & $63(63)$ & $66(66)$ & 0.768 \\
\hline Secondary & $37(37)$ & $34(34)$ & \\
\hline Prior pelvic surgery & $22(22)$ & 19 (19) & 0.726 \\
\hline Oligomenorrhea & $65(65)$ & $63(63)$ & 0.883 \\
\hline Acne & $22(22)$ & $24(24)$ & 0.867 \\
\hline Hirsutism & $45(45)$ & $42(42)$ & 0.776 \\
\hline Waist to hip ratio & $0.83 \pm 0.09$ & $0.84 \pm 0.08$ & 0.935 \\
\hline HOMA-IR & $2.8 \pm 0.47$ & $2.5 \pm 0.52$ & 0.669 \\
\hline Basal FSH (mIU/ml) & $4.8 \pm 0.19$ & $5.2 \pm 0.17$ & 0.119 \\
\hline Basal LH (mIU/ml) & $6.9 \pm 0.36$ & $7.3 \pm 0.28$ & 0.382 \\
\hline Testosterone (nmol/l) & $0.042 \pm 0.002$ & $0.045 \pm 0.001$ & 0.19 \\
\hline Sperm count (million/ml) & $51.2 \pm 1.99$ & $53.4 \pm 2.14$ & 0.451 \\
\hline Total sperm motility $(\%)$ & $64.7 \pm 0.94$ & $64.2 \pm 1.07$ & 0.726 \\
\hline Progressive sperm motility (\%) & $44.3 \pm 0.82$ & $43.7 \pm 0.93$ & 0.624 \\
\hline
\end{tabular}




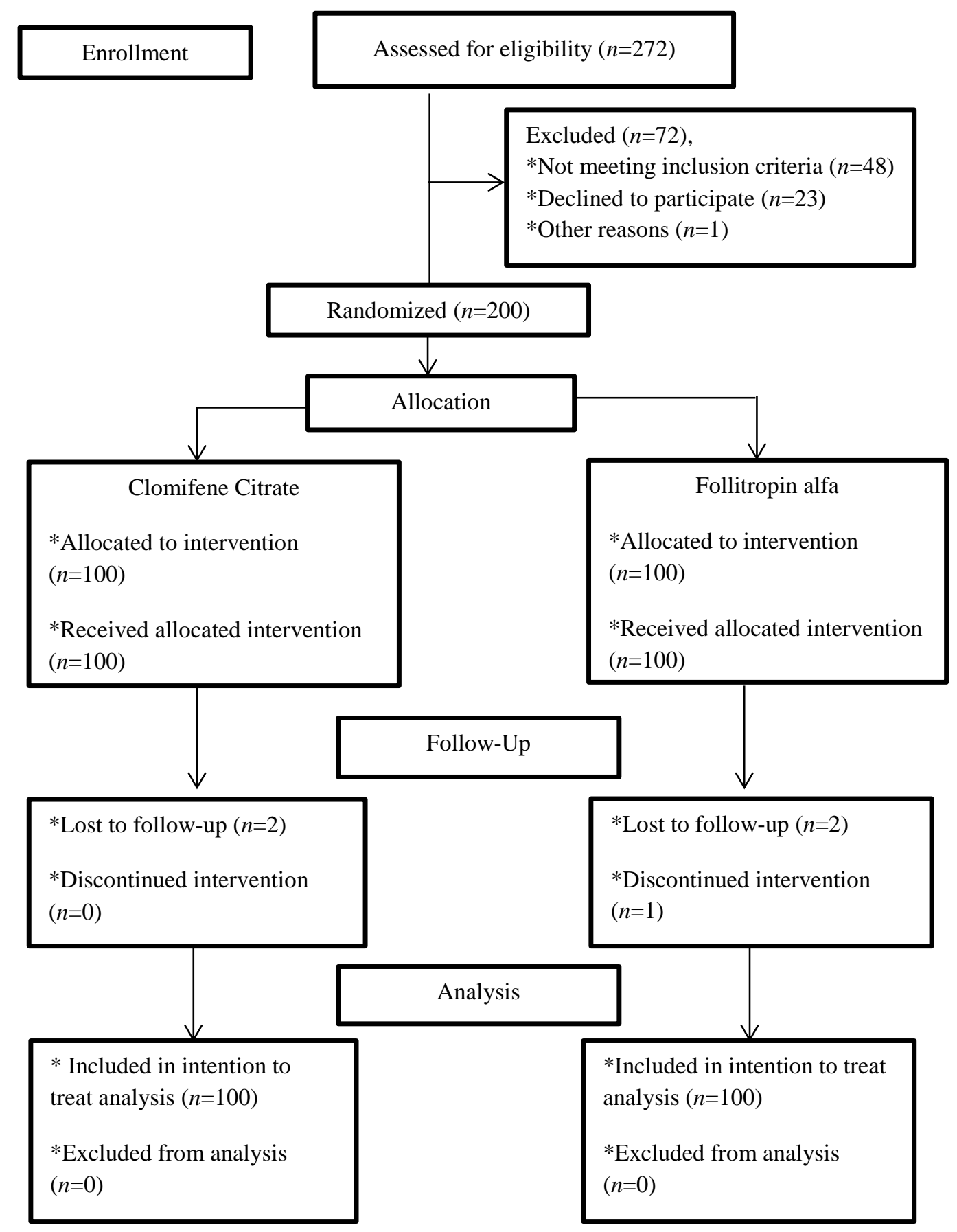

Figure 1: Patients flow chart, CONSORT.

Table 2 shows that there was no statistically significant difference between the two study groups regarding ovulation rate, endometrial thickness, serum estradiol on the day of HCG injection, and mid-luteal serum progesterone level. The conception rates in both groups were similar. The biochemical pregnancy rate was $21 \%$ in the clomifene group compared with $24 \%$ in the follitropin-alfa group $[P=0.735 ; \mathrm{RR}=0.88,(95 \% \mathrm{CI}, 0.52-$ 1.47)]. As well, the clinical pregnancy rate was $19 \%$ in the clomifene group compared with $21 \%$ in the follitropin- alfa group $[P=0.86 ; \mathrm{RR}=0.9$, (95\% CI, 0.52-1.58)]. As regards the adverse drug events there was no statistically significant difference between the two groups concerning OHSS, gastrointestinal upset, or flushing (Table 2). Cases with OHSS in both groups (one in the CC group and three in the gonadotropins group) were of the mild form. All cases of OHSS were managed conservatively as an outpatient in the Infertility Clinic and none of them got pregnant. 
Table 2: Outcomes measures.

\begin{tabular}{|c|c|c|c|c|}
\hline Variable & CC group $(n=100)(\%)$ & $\begin{array}{l}\text { Follitropin-alfa } \\
\text { group }(\boldsymbol{n}=\mathbf{1 0 0})(\%)\end{array}$ & $P$ value & $\mathbf{R R}(\mathbf{9 5} \% \mathbf{C I})$ \\
\hline $\begin{array}{l}\text { Patients with mature } \\
\text { follicles }(\geq 16 \mathrm{~mm})\end{array}$ & $51(51)$ & $56(56)$ & 0.571 & $0.91(0.7-1.18)$ \\
\hline Endometrial thickness & $9.1 \pm 0.29$ & $9.4 \pm 0.31$ & 0.766 & \\
\hline $\begin{array}{l}\text { Estradiol level on day of } \\
\text { HCG injection (pmol/l) }\end{array}$ & $4274.1 \pm 240.5$ & $4459.2 \pm 228$ & 0.577 & \\
\hline $\begin{array}{l}\text { Patients with progesterone } \\
\geq 30 \mathrm{nmol} / 1\end{array}$ & $46(46)$ & $49(49)$ & 0.777 & $0.94(0.7-1.26)$ \\
\hline Biochemical pregnancy & $21(21)$ & $24(24)$ & 0.735 & $0.88(0.52-1.47)$ \\
\hline Clinical pregnancy & $19(19)$ & $21(21)$ & 0.86 & $0.9(0.52-1.58)$ \\
\hline \multicolumn{5}{|l|}{ Adverse drug events } \\
\hline OHSS & $1(1)$ & $3(3)$ & 0.621 & $0.33(0.04-3.15)$ \\
\hline GIT discomfort & $5(5)$ & $1(1)$ & 0.212 & $5(0.6-42)$ \\
\hline Flushing & $2(2)$ & 0 & 0.498 & \\
\hline
\end{tabular}

\section{DISCUSSION}

Ovulation induction in women with PCOS who present with CC resistant anovulatory infertility remains a major challenge in gynecologic endocrinology. ${ }^{15}$ Classical alternatives for CC-resistant patients include gonadotropins therapy and laparoscopic ovarian diathermy. However, due to the cost and risk inherent in these therapies, alternative treatments are attractive. ${ }^{16}$ In this randomized trial, we have evaluated the effectiveness of prolonged use of CC for 10 days as a first line method of ovulation induction in PCOS women with $\mathrm{CC}$ resistant in comparison with follitropin-alfa. Both treatment strategies provide comparable outcomes. The biochemical pregnancy rate was $21 \%$ in the CC group, and $24 \%$ in the follitropin-alfa group $[P=0.735 ; R R=0.88$, (95\% CI, 0.52-1.47)]. Similarly, the clinical pregnancy rate was $19 \%$ in the CC group, and $21 \%$ in the follitropinalfa group $[P=0.86 ; \quad R R=0.9, \quad(95 \%$ CI, 0.52-1.58)]. Furthermore, no differences have been detected between both groups in the ovulation rate or the adverse drug events.

Though numerous studies and reviews have evaluated CC resistance in patients with $\mathrm{PCOS}$, yet the data focusing on the etiology behind the occurrence of $\mathrm{CC}$ resistance are still lacking. Indeed, the major manifestations of PCOS include menstrual cycle irregularities due to anovulation and signs of androgen excess. ${ }^{17}$ A proposed mechanism for anovulation in PCOS patients is that, under the increased stimulatory effect of LH secreted by the anterior pituitary, encouragement of the ovarian theca cells activity is increased. In turn, theca cells increase the production of androgens. As there is a decreased level of FSH relatively to $\mathrm{LH}$, the ovarian granulosa cells would not aromatize the androgens to estrogens, which result in decreased estrogen levels and subsequent anovulation. ${ }^{18}$ On the other hand, the effectiveness of $\mathrm{CC}$ in induction of ovulation is mainly attributed to actions at the level of the hypothalamus. Diminution of hypothalamic estrogen receptors precludes correct interpretation of circulating estrogen levels. The reduced levels of estrogen feedback generate normal compensatory mechanisms that alter pulsatile hypothalamic gonadotropin-releasing hormone (GnRH) secretion to motivate increased pituitary gonadotropin release that consequently stimulate ovarian follicular activity. ${ }^{6}$ In ovulatory women, CC treatment elevates GnRH pulse frequency. ${ }^{19}$ Women with PCOS have an anovulation with abnormally high $\mathrm{GnRH}$ pulse frequency. CC treatment, in anovulatory women with PCOS, increases pulse amplitude but not frequency. ${ }^{20}$ During CC treatment, the levels of both LH and FSH rise, then they are falling again after the typical 5-day course of therapy is completed. ${ }^{21}$ If this FSH rise fulfilled the requirements of the leading follicle/s they will be pushed through the final part of their growth progression till ovulation. $^{22}$ It has been suggested that the duration of FSH rise is more important than magnitude for follicular development. $^{23}$ In successful CC treatment cycles; follicular growth occurs in parallel with increasing serum estrogen, eventually prompting LH surge and ovulation. It has been advocated that FSH levels are maintained high with the 10 day protocol denoting that the hypothalamus remains responsive to $\mathrm{CC}$ for up to that duration. $^{24}$

Previous studies that assessed follitropin-alfa for ovulation induction in women with CC resistant PCOS, have reported that the pregnancy rate per stimulated cycle was approximately $18-20 \% .^{8,25-27}$ However, the current trial revealed comparable biochemical and clinical pregnancy rates $(21 \%$ and $19 \%$ respectively) upon using $\mathrm{CC}$ for 10 days in ovulation induction for CC resistant Patients. Furthermore, our data emphasized that the pregnancy rates of CC 10 day protocol were similar to follitropin-alfa therapy. Our data were consistent with 
Fluker et al. $^{28}$ that conducted a retrospective study to evaluate the $\mathrm{CC} 10$ day protocol in $\mathrm{CC}$ resistant patients and reported $47 \%$ ovulation rate and $17 \%$ pregnancy rate. Furthermore, a more recent randomized controlled trial by Badawy et al. established that the extended $\mathrm{CC}$ regimen resulted in modest ovulation and pregnancy rates (28.1\%, 11.4\%; respectively), and it deserved the trial before other expensive and more sophisticated alternatives. $^{29}$

In conclusion, prolonging the duration of $\mathrm{CC}$ for 10 days is as efficient as follitropin-alfa in inducing ovulation, and achieving pregnancy in PCOS patients with $\mathrm{CC}$ resistance. However, $\mathrm{CC}$ is cheap, easy to administer, and has rare side-effects. It can be used as a first line management for $\mathrm{CC}$ resistance before other alternatives. Further larger multi-centric studies are required to confirm our data and to provide a more powerful conclusion.

\section{Funding: No funding sources}

Conflict of interest: None declare

Ethical approval: The study was approved by the local institutional ethics committee

\section{REFERENCES}

1. Teede H, Deeks A, Moran L. Polycystic ovary syndrome: A complex condition with psychological, reproductive and metabolic manifestations that impacts on health across the lifespan. BMC Med. 2010;8:41.

2. Amer SA, Li TC, Metwally M, Emarh M, Ledger WL. Randomized controlled trial comparing laparoscopic ovarian diathermy with clomiphene citrate as a first-line method of ovulation induction in women with polycystic ovary syndrome. Hum Reprod. 2009;24:219-25.

3. Brown J, Farquhar C, Beck J, Boothroyd C, Hughes E. Clomiphene and anti-estrogens for ovulation induction in PCOS. Cochrane Database Syst Rev. 2009;4:CD002249.

4. Saha L, Kaur S, Saha PK. N-acetyl cysteine in clomiphene citrate resistant polycystic ovary syndrome: A review of reported outcomes. J Pharmacol Pharmacother. 2013;4:187-91.

5. Overbeek A, Kuijper EA, Hendriks ML, Blankenstein MA, Ketel IJ, Twisk JW et al. Clomiphene citrate resistance in relation to folliclestimulating hormone receptor Ser680Serpolymorphism in polycystic ovary syndrome. Hum Reprod. 2009;24:2007-13.

6. Practice Committee of the American Society for Reproductive Medicine. Use of clomiphene citrate in infertile women: a committee opinion. Fertil Steril. 2013;100:341-8.

7. Barontini M, Garc1'a-Rudaz MC, Veldhuis JD. Mechanisms of hypothalamic-pituitary-gonadal disruption in polycystic ovarian syndrome. Arch Med Res. 2001;32:544-52.
8. Thessaloniki ESHRE/ASRM-Sponsored PCOS Consensus Workshop Group. Consensus on infertility treatment related to polycystic ovary syndrome. Fertil Steril. 2008;89:505-22.

9. Revelli A, Poso F, Gennarelli G, Moffa F, Grassi G, Massobrio M. Recombinant versus highly-purified, urinary follicle-stimulating hormone (r-FSH vs. HP$\mathrm{uFSH}$ ) in ovulation induction: A prospective, randomized study with cost-minimization analysis. Reprod Biol Endocrinol. 2006;4:38.

10. Palomba S, Falbo A, Zullo F. Management strategies for ovulation induction in women with polycystic ovary syndrome and known clomifene citrate resistance. Curr Opin Obstet Gynecol. 2009;21:46573.

11. Fauser BC, Tarlatzis BC, Rebar RW, Legro RS, Balen AH, Lobo R et al. Consensus on women's health aspects of polycystic ovary syndrome (PCOS): The Amsterdam ESHRE/ASRM-sponsored $3^{\text {rd }}$ PCOS consensus workshop group. Fertil Steril. 2012;97:28-38.

12. Matthews DR, Hosker JP, Rudenski AS, Naylor BA, Treacher DF, Turner RC. Homeostasis model assessment: Insulin resistance and beta-cell function from fasting plasma glucose and insulin concentrations in man. Diabetologia. 1985;28:412-9.

13. The Practice Committee of the American Society for Reproductive Medicine. Diagnostic evaluation of the infertile male: A committee opinion. Fertil Steril. 2012;98:294-301.

14. Mukherjee S, Sharma S, Chakravarty BN. Comparative evaluation of pregnancy outcome in gonadotrophin-clomiphene combination vs. clomiphene alone in polycystic ovarian syndrome and unexplained infertility - A prospective clinical trial. J Hum Reprod Sci. 2010;3:80-4.

15. Amsterdam ESHRE/ASRM-Sponsored 3rd PCOS Consensus Workshop Group. Consensus on women's health aspects of polycystic ovary syndrome (PCOS). Hum Reprod. 2012;27:14-24.

16. Nahuis MJ, Oude Lohuis E, Kose N, Bayram N, Hompes P, Oosterhuis GJ et al. Long-term follow-up of laparoscopic electrocautery of the ovaries versus ovulation induction with recombinant FSH in clomiphene citrate-resistant women with polycystic ovary syndrome: An economic evaluation. Hum Reprod. 2012;27:3577-82.

17. Panidis D, Tziomalos K, Papadakis E, Kandaraki EA, Katsikis I. The guidelines issued by the European Society for Human Reproduction and Embryology and the American Society for Reproductive Medicine regarding the induction of ovulation with metformin in patients with the polycystic ovary syndrome potentially require reconsideration. Hormones. 2013;12:192-200.

18. Dunaif A, Wu X, Lee A, Diamanti-Kandarakis E. Defects in insulin receptor signaling in vivo in the polycystic ovary syndrome (PCOS). Am J Physiol Endocrinol Metab. 2001;281:E392-9. 
19. Kerin JF, Liu JH, Phillipou G, Yen SS. Evidence for a hypothalamic site of action of clomiphene citrate in women. J Clin Endocrinol Metab 1985;61:265-8.

20. Kettel LM, Roseff SJ, Berga SL, Mortola JF, Yen SS. Hypothalamic pituitaryovarian response to clomiphene citrate in women with polycystic ovary syndrome. Fertil Steril. 1993;59:532-8.

21. Rebar R, Judd HL, Yen SSC, Rakoff J, VandenBerg $\mathrm{G}$, Naftolin F. Characterization of the inappropriate gonadotropin secretion in polycystic ovary syndrome. J Clin Invest. 1976;57:1320-9.

22. Schoemaker J, van Weissenbruch MM, Scheele F, van der Meer M. The FSH threshold concept in clinical ovulation induction. Balliere Clin Obstet Gynecol. 1993;7:297-308.

23. Schipper I, Hop WC, Fauser BC. The folliclestimulating hormone (FSH) threshold/window concept examined by different interventions with exogenous FSH during the follicular phase of the normal menstrual cycle: Duration, rather than magnitude, of FSH increase affects follicle development. J Clin Endocrinol Metab. 1998;83:1292-8.

24. Messinis IE. Ovulation induction: A mini review. Hum Reprod. 2005;20:2688-97.
25. Christin-Maitre S, Hugues JN; Recombinant FSH Study Group. A comparative randomized multicentric study comparing the step-up versus stepdown protocol in polycystic ovary syndrome. Hum Reprod. 2003;18:1626-31.

26. Bruna-Catalán I, Menabrito M. Ovulation induction with minimal dose of follitropin alfa: A case series study. Reprod Biol Endocrinol .2011;24;9:142.

27. Nyboe Andersen A, Balen AH, Platteau P, Pettersson G, Arce JC. Prestimulation parameters predicting live birth in anovulatory WHO Group II patients undergoing ovulation induction with gonadotrophins. Hum Reprod. 2010;25:1988-95.

28. Fluker MR, Wang IY, Rowe TC. An extended 10day course of clomiphene citrate (CC) in women with CC-resistant ovulatory disorders. Fertil Steril. 1996;66:761-4.

29. Badawy A, Allam A, Abulatta M. Extending clomiphene treatment in clomiphene-resistant women with PCOS: a randomized controlled trial. Reprod Biomed Online 2008;16(6):825-9.

DOI: $10.5455 / 2320-1770 . i j r \operatorname{cog} 20140303$

Cite this article as: Hassan MF. Efficacy of extended clomifene citrate regimen in comparison with gonadotropins in clomifene citrate-resistant women with polycystic ovary syndrome. Int $\mathbf{J}$ Reprod Contracept Obstet Gynecol 2014;3:19-25. 